\title{
Effect of the COVID-19 Pandemic on Cost-Related Prescription Nonredemption, Health care Seeking and Hospitalization in Hungary: A Nationally Representative Cross-Sectional Study
}

\author{
Bayu Begashaw Bekele ${ }^{1,2,3}$, Bahaa Aldin Alhaffar ${ }^{4}$, Rahul Naresh Wasnik ${ }^{1,3}$, János Sándor ${ }^{3 *}$ \\ ${ }^{1}$ Doctoral School of Health Sciences, University of Debrecen, 4028 Debrecen, Hungary; ba- \\ yu.begashaw@med.unideb.hu or baybeg121@gmail.com (B.B.B.); rahul.naresh@med.unideb.hu (R.N.W.) \\ 2 Department of Public Health, College of Health Sciences, Mizan Tepi University, 260 Mizan Aman, Ethiopia; \\ ${ }^{3}$ Department of Public Health and Epidemiology, Faculty of Medicine, University of Debrecen, 4028 Debrecen, \\ Hungary; janos sandor @med.unideb.hu (S.J.) \\ ${ }^{4}$ Faculty of public health, University of Debrecen, 4028, Debrecen, Hungary; bhaa.alhafar@gmail.com (B.A.A.) \\ * Correspondence: sandor.janos@med.unideb.hu ; Tel.: +36 209323140
}

\begin{abstract}
Background: Although, negative repercussions of inadequate health service use on the health outcomes has been presumably exacerbated by COVID-19, the impact of the pandemic measures has been not evaluated properly yet. Objective: Our study aimed to quantify the COVID-19 pandemic measures' effect on the general practitioner (GP) visit, specialist care, hospitalization and cost-related prescription nonredemption (CRPNR) among adults in Hungary, and to identify the social strata susceptible to the pandemic effect. Methods: This community-based cross-sectional study based on nationally representative data of 6,611 (Nprepandemic=5,603 and Npandemic $=1,008$ ) subjects aged 18 years and above. Data were obtained from the European Health Interview Survey 2019 (EHIS) and International Social Survey Program 2021 (ISSP) for prepandemic and pandemic, respectively. Multivariable logistic regression models were applied to determine the sociodemographic and clinical factors influencing the health care use by odds ratios (OR) along with the corresponding 95\% confidence intervals (CI). To identify the social strata susceptible to pandemic effect, the interaction of the time of data collection with level of education, marital status, and ethnicity, was also tested. Results: While, the CRPNR did not changed, the frequency of GP visit, specialist care and hospitalization rate were remarkably reduced by $22.2 \%$, $26.4 \%$, and $6.7 \%$, respectively, during the pandemic in Hungary. Roma proved to be not specifically affected by the pandemic in any studied respect. The pandemic restructuring of health care impacted the social subgroups evenly with respect to hospital care. However, the pandemic effect was weaker among primary educated adults (ORhigh-school vs primary-education $=0.434 ; 95 \% \mathrm{CI}$ 0.243-0.776, ORhigh-school vs primary-education $=0.598$; 95\% CI 0.364-0.985), and among widows (ORwidowed vs married $=2.284 ; 95 \%$ CI 1.043-4.998, ORwidowed vs married=1.915; 95\% CI 1.157-3.168) on the frequency of GP visit and specialist visit; and the prepandemic CRPNR inequality by level of education was increased (ORhigh-school vs primary-education $=0.236$; 95\% CI 0.075-0.743). Conclusion: Primary educated and widowed did not follow the general trend, and their prepandemic limited health care use was not reduced further during pandemic, resulting in an inequality reduction. The vulnerability of primary educated to CRPNR was the only gap widened in the pandemic period. This shows that although the management of pandemic health care use restrictions was implemented by increasing the social inequality in Hungary, the prevention of inequity in drug availability for primary educated individuals could require more support.
\end{abstract}

Keywords: CRPNR; COVID-19 pandemic; hospital admission; GP visit; Hungary; interaction effect; Roma; specialist care 


\section{Introduction}

Inequalities in health care utilization are a major global public health issue. Thematic determinants of the problem can be pre-existing, enabling and need-based [1]. The pre-existing features are sociodemographic characteristics, such as age, sex, ethnicity, residence, marital status and social relationships. Enabling factors include logistic perspectives, education level, economic potential, accessibility, quality of service delivery, and the availability of drugs and medical supplies. Need-based factors are the most immediate ones (perceived general health status, nature or duration of the disease, intensity of the pain of illness), which push individuals to seek health care utilization [2-5]. Altogether, these determinants could appear as either facilitators or inhibitors to attain services and lead to multifaceted corresponding health consequences. The pessimistic consequences of less service utilization could be further complications, a poor prognosis, disability and death accompanied by further health care costs [6-9].

The coronavirus disease 2019 (COVID-19) pandemic, necessary health services restructuring and lockdown restriction misery that started in March 2020 harshly reduced health care service utilization among beneficiaries [10]. Face-to-face visits to primary and secondary health care and elective hospital admissions were forgone, postponed or declined during the lockdown [11]. The pandemic made things uncertain among patients regarding their economy, health, society, behavior, social and daily lives, and livelihood $[10,12-16]$. In the pandemic period, many people lost their jobs, income, access to health care, compliance and trust in health care [17-19]. These factors hindered health care institution visits and admission, even for severe medical cases during the pandemic [10,2027].

The hospital admission rate fell 39\%, and GP and specialist visits fell by $6 \%$ following the lockdown in Germany [10]. Similarly, the hospital admission incidence was reduced by $22 \%$ in Croatia [28], and basic and upgraded life-saving services were downgraded by $7 \%$ in Finland [16]. The GP and specialist visits postponed due to the lockdown were the most frequent in Portugal (55\%) and reached their lowest in Bulgaria $(2 \%)$, with an EU average of $26 \%$. However, patients who missed treatments due to fear of acquiring the COVID infection during the lockdown were the highest in Israel (27\%) and the lowest in Slovenia and Spain (4\%), with an EU average of 12\% [29]. Although it is known that most EU countries did not modify out-of-pocket payments for medication during the pandemic lockdown [11], the pandemic impact on medication has not yet been reported.

There are only a few studies published so far about the health care utilization of vulnerable social groups during the pandemic. A study from South Korea showed that skipping primary health care services during the pandemic lockdown was higher among married than single/separated/divorced subgroups, but the pandemic lockdown did not result in variations for not utilizing health care services across the education stratum [30]. In the United Kingdom (UK), patients with all kinds of physical and mental cases showed considerable decreases in primary care contact during the first lockdown [31]. Additionally, another study from the UK revealed that ethnic minorities were more vulnerable in terms of emergency department and referral unit visits to the pandemic compared to their reference subcategories [32]. Moreover, according to the European Union Agency for Fundamental Rights, the health risk of the Roma population during the pandemic lockdown left them vulnerable and unable to access health care across Europe [33]. A pooled analysis of ethnic vulnerability due to the first wave pandemic lockdown revealed that the hospitalization rate was higher for ethnic minorities in the US and Europe. Hispanics, Asians and Black African-Americans were 2.08, 1.59 and 1.53 times more likely to be hospitalized than Whites [34].

In the context of Hungary, due to the increased number of new COVID-19 infections in the first wave of the epidemic, the lockdown was implemented from March to June 
2020 in Hungary. Similarly, the second and third pandemic restrictions were applied from November 2020 and continued until now with some easing of the regulations [35]. The pandemic measures included regulated restrictions of primary, secondary, and tertiary health services to ensure the capacities for COVID-19 patients' care and vaccination programs, but the lockdown restrictions were not applicable for pharmaceuticals and emergency health care services. However, Hungary is not special in Europe with respect to the effectiveness of pandemic control, as reflected in the excess mortality data [36]. Another study revealed that sociodemographic inequity highly determined the impact of the pandemic in Hungary. The most deprived settings had a lower incidence of morbidity with higher mortality and case fatality rates [37]. Nonetheless, we did not find any investigation of the dynamics of fundamental health care service utilization attributed to the pandemic lockdown in Hungary. Furthermore, cost-related prescription nonredemption (CRPNR) and the pandemic impact have not yet been studied.

Therefore, we found that it is indispensable to investigate the effect of the COVID-19 pandemic together with other explanatory characteristics on GP visits, specialist visits, hospitalizations and CRPNR in Hungary, which has not been evaluated in previous studies. In detail, we (1) scrutinized the prevalence of GP visits, specialist visits, hospitalizations and CRPNR before and during the lockdown of the COVID-19 pandemic, (2) investigated the effect of the pandemic on CRPNR GP visits, specialist visits and hospitalizations controlled for established predictors, and (3) determined subgroups susceptible to GP visits, specialist visits, hospitalizations and CRPNR elicited by the pandemic.

\section{Materials and Methods}

\subsection{Setting}

This study is a population-based, comparative cross-sectional investigation. Data for analysis were obtained from the 2021 International Social Survey Program (ISSP) [38] and the 2019 European Health Interview Survey (EHIS) Wave 3 databases of Hungary [39]. Both surveys were based on a representative sample for the whole country, and both collected data on health care use over a one-year retrospective period.

\subsubsection{Before the COVID-19 pandemic data source}

To describe the outcome parameters (i.e., the frequency of GP visits, specialist visits, hospitalization, and CRPNR) and the pertinent characteristics among adults before the pandemic, we used the third wave of the 2019 EHIS dataset of Hungary. It contained four major thematic modules on health status, health care use, lifestyle, and sociodemographic status. Data were collected by personal interviews from September 2019 to January 2020. The detailed techniques were published elsewhere [39]. The data were obtained from a representative sample of 5,603 participants aged 18 years old and above.

\subsubsection{The data source during the COVID-19 pandemic}

We used data from the Health and Health Care II panel of ISSP in 2021 to describe the health care use indicators with their determinants during the COVID-19 pandemic. The methods used for this survey were published previously [40]. This study was conducted from March 15 to May 30, 2021, during the lockdown of the third wave of COVID-19. A representative sample of 1,008 Hungarian adults 18 years old and above was randomly selected and interviewed in this study.

\subsection{Outcome variables}

The GP visit in a year and specialist visits in a year were dichotomous variables for subjects who had a history of visiting their GPs and specialists, respectively, in the last 12 months prior to the survey. Similarly, hospital admission in a year was a dichotomous variable if the patient had stayed at least one night in a hospital in the last 12 months before the survey. CRPNR was a dichotomous variable defined as the respondents hav- 
ing missed, skipped, or replaced a prescribed drug due to financial problems at least once in the last 12 months.

\subsection{Explanatory variables}

The primary explanatory variable in this study was the time of data collection. Participants representing prepandemic and pandemic conditions were distinguished. Outcome variables were determined for the year before the first wave of the COVID-19 pandemic by EHIS and for the 12-month period affected by the pandemic.

Education was a variable with four categories (completed grade 8 primary school, attended vocational school without a high school diploma, high school graduation with a diploma, and tertiary education included college and university graduates). The region was classified as the residential place of the subjects as Central-Hungary, Central-Transdanubia, Western-Transdanubia, Southern-Transdanubia, Northern-Hungary, Northern-Great-Plain and Southern-Great-Plain. The marital status of the participants was classified as married, single, widowed, and divorced. According to the self-reported ethnicity, the Roma and the non-Roma were distinguished (the Roma is the only large ethnic minority group in Hungary. They comprise $8.8 \%$ of the population [41]).

The age of the subjects was categorized as 18-34, 35-64 and 65 years and above. Sex was classified as male or female. The most prevalent chronic diseases, such as chronic obstructive pulmonary disease (COPD), ischemic heart disease (IHD), hypertension, diabetes mellitus, and malignancy, were registered by self-declaration of the participants as dichotomous variables.

\subsection{Statistical analysis}

The dataset obtained by merging the two surveys was analyzed using SPSS version 21. Ethical approval was not required for the secondary analysis of the anonymized data.

Bivariate analysis was performed to assess the association between the independent and outcome variables by applying logistic regression analyses. Then, multivariable logistic regression models were applied to control the confounding effect of socioeconomic status indicators (education, marital status and ethnicity) on the outcome variables after adjusting for other independent determinants (age, region, sex, COPD, IHD, diabetes, hypertension, malignancy) of each outcome variable. Furthermore, the interaction between the time of data collection (distinguishing prepandemic and pandemic periods) and the socioeconomic status indicators were included in the model. The GP visits, specialist visits, hospital admissions, and CRPNR within a year were the outcome variables in the four applied logistic regression models. The aim of testing for interactions was to identify the social groups vulnerable to the detrimental effect of the pandemic. The results are reported in terms of odds ratios (ORs), adjusted odds ratios (aORs), and odds ratios for interactions with time (iORs) with the corresponding $95 \%$ confidence intervals (CIs).

\section{Results}

The merged sample of this study consisted of 6611 adults. Records of participants who had no responses for corresponding outcomes were removed from the database. After cleaning for missing values for outcome variables, four distinct datasets were prepared. The sample sizes for the analysis of GP visits, specialist visits, hospital admissions and CRPNR in a year were $6370\left(\mathrm{~N}_{\text {prepandemic }}=5368 ; \mathrm{N}_{\text {pandemic }}=1002\right), 6317$ ( $\mathrm{N}_{\text {prepandemic }}=5323$; $\left.\mathrm{N}_{\text {pandemic }}=994\right), 6408\left(\mathrm{~N}_{\text {prepandemic }}=5408 ; \mathrm{N}_{\text {pandemic }}=1000\right)$, and $5028\left(\mathrm{~N}_{\text {prepandemic }}=4337 ; \mathrm{N}_{\text {pandem- }}\right.$ ic=691), respectively.

\subsection{Sociodemographic and clinical characteristics}


The sociodemographic composition of the prepandemic and pandemic samples showed statistically significant differences. There was an overrepresentation of middle-aged individuals, women, adults with vocational or high school-level education, and Roma individuals in each pandemic sample, and furthermore, Central Hungarian residents with hospital visits and CRPNR samples, and divorced individuals in each sample apart from the GP visit dataset in the pandemic sample, were detected. Concerning the clinical factors, the representation of patients with diabetes and hypertension was different between the two study periods. (Table 1) 
Table 1. Sociodemographic and clinical characteristics of studied samples (number of cases and percentages by subgroups) in pre-pandemic and pandemic periods.

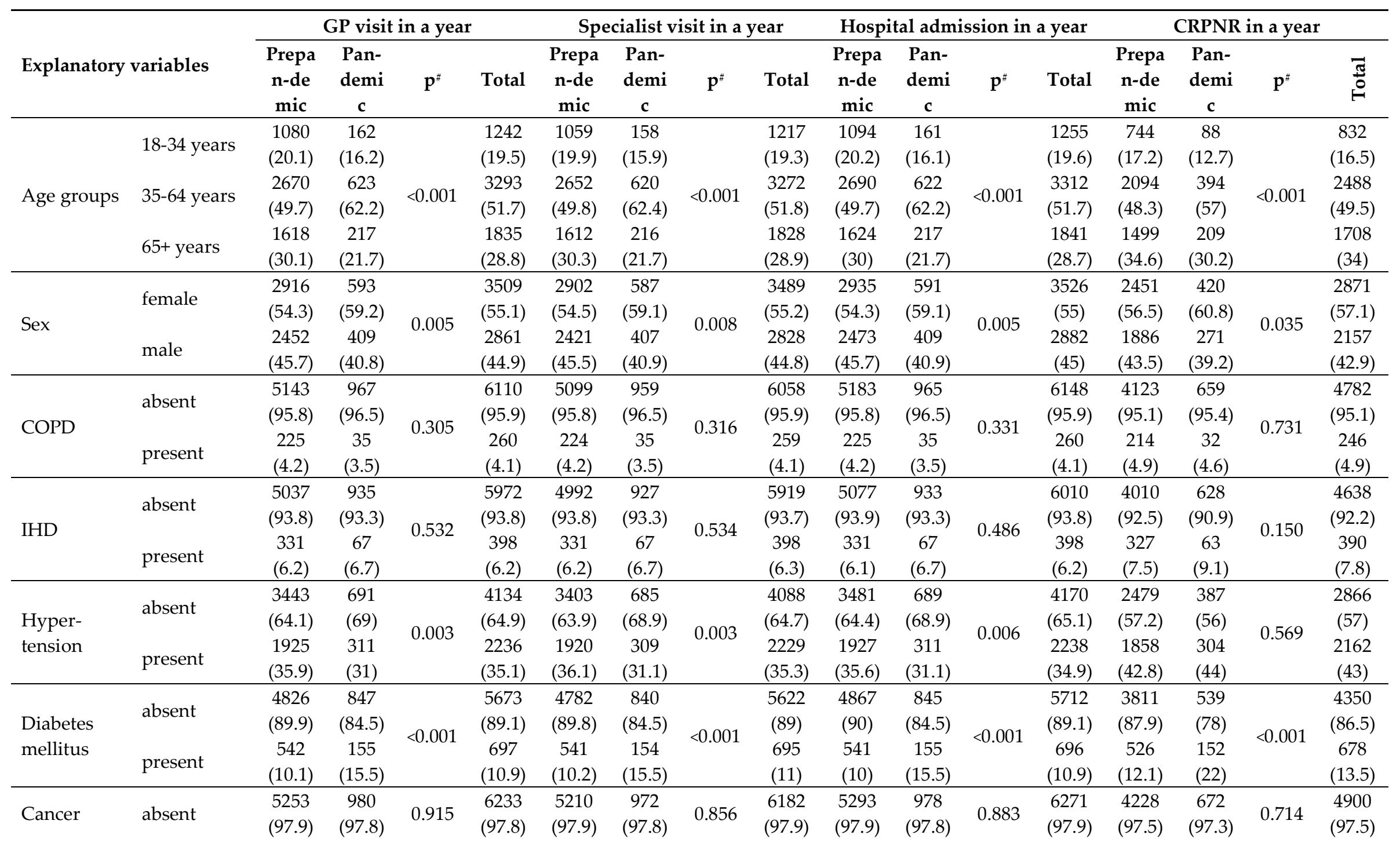




\begin{tabular}{|c|c|c|c|c|c|c|c|c|c|c|c|c|c|c|c|c|c|}
\hline & present & $\begin{array}{l}115 \\
(2.1) \\
\end{array}$ & $\begin{array}{c}22 \\
(2.2) \\
\end{array}$ & & $\begin{array}{l}137 \\
(2.2)\end{array}$ & $\begin{array}{l}113 \\
(2.1)\end{array}$ & $\begin{array}{c}22 \\
(2.2) \\
\end{array}$ & & $\begin{array}{l}135 \\
(2.1) \\
\end{array}$ & $\begin{array}{l}115 \\
(2.1) \\
\end{array}$ & $\begin{array}{c}22 \\
(2.2) \\
\end{array}$ & & $\begin{array}{l}137 \\
(2.1)\end{array}$ & $\begin{array}{l}109 \\
(2.5)\end{array}$ & $\begin{array}{c}19 \\
(2.7)\end{array}$ & & $\begin{array}{l}128 \\
(2.5)\end{array}$ \\
\hline \multirow{10}{*}{ Region } & $\begin{array}{l}\text { Central-Hu } \\
\text { ngary }\end{array}$ & $\begin{array}{l}1533 \\
(28.6)\end{array}$ & $\begin{array}{c}304 \\
(30.3)\end{array}$ & \multirow{10}{*}{0.334} & $\begin{array}{l}1837 \\
(28.8)\end{array}$ & $\begin{array}{l}1523 \\
(28.6)\end{array}$ & $\begin{array}{c}304 \\
(30.6)\end{array}$ & \multirow{10}{*}{0.289} & $\begin{array}{l}1827 \\
(28.9)\end{array}$ & $\begin{array}{l}1549 \\
(28.6)\end{array}$ & $\begin{array}{c}304 \\
(30.4)\end{array}$ & \multirow{10}{*}{$<0.001$} & $\begin{array}{l}1853 \\
(28.9)\end{array}$ & $\begin{array}{l}1242 \\
(28.6)\end{array}$ & $\begin{array}{c}202 \\
(29.2)\end{array}$ & \multirow{10}{*}{$<0.001$} & $\begin{array}{l}1444 \\
(28.7)\end{array}$ \\
\hline & Central-Tra & 593 & 110 & & 703 & 590 & 110 & & 700 & 595 & 110 & & 705 & 465 & 89 & & 554 \\
\hline & & (11) & (11) & & (11) & (11.1) & (11.1) & & (11.1) & (11) & (11) & & (11) & (10.7) & (12.9) & & (11) \\
\hline & Northern-G & 836 & 148 & & 984 & 829 & 146 & & 975 & 840 & 148 & & 988 & 667 & 102 & & 769 \\
\hline & reat-Plain & (15.6) & (14.8) & & (15.4) & (15.6) & (14.7) & & (15.4) & (15.5) & (14.8) & & (15.4) & (15.4) & (14.8) & & (15.3) \\
\hline & $\begin{array}{l}\text { Northern-H } \\
\text { ungary }\end{array}$ & $\begin{array}{c}673 \\
(12.5)\end{array}$ & $\begin{array}{l}120 \\
(12)\end{array}$ & & $\begin{array}{c}793 \\
(12.4)\end{array}$ & $\begin{array}{c}663 \\
(12.5)\end{array}$ & $\begin{array}{c}115 \\
(11.6)\end{array}$ & & $\begin{array}{c}778 \\
(12.3)\end{array}$ & $\begin{array}{c}678 \\
(12.5)\end{array}$ & $\begin{array}{c}119 \\
(11.9)\end{array}$ & & $\begin{array}{c}797 \\
(12.4)\end{array}$ & $\begin{array}{l}562 \\
(13)\end{array}$ & $\begin{array}{c}76 \\
(11)\end{array}$ & & $\begin{array}{c}638 \\
(12.7)\end{array}$ \\
\hline & Southern-G & 660 & 133 & & 793 & 657 & 133 & & 790 & 666 & 132 & & 798 & 545 & 97 & & 642 \\
\hline & reat-Plain & (12.3) & (13.3) & & (12.4) & (12.3) & (13.4) & & (12.5) & (12.3) & (13.2) & & (12.5) & (12.6) & (14) & & (12.8) \\
\hline & $\begin{array}{l}\text { Southern-Tr } \\
\text { ansdanubia }\end{array}$ & $\begin{array}{l}508 \\
(9.5)\end{array}$ & $\begin{array}{c}86 \\
(8.6)\end{array}$ & & $\begin{array}{l}594 \\
(9.3)\end{array}$ & $\begin{array}{l}502 \\
(9.4)\end{array}$ & $\begin{array}{c}86 \\
(8.7)\end{array}$ & & $\begin{array}{l}588 \\
(9.3)\end{array}$ & $\begin{array}{l}513 \\
(9.5)\end{array}$ & $\begin{array}{c}86 \\
(8.6)\end{array}$ & & $\begin{array}{l}599 \\
(9.3)\end{array}$ & $\begin{array}{l}413 \\
(9.5)\end{array}$ & $\begin{array}{c}53 \\
(77)\end{array}$ & & $\begin{array}{l}466 \\
(9.3)\end{array}$ \\
\hline & $\begin{array}{l}\text { Western-Tr } \\
\text { ansdanubia }\end{array}$ & $\begin{array}{c}565 \\
(10.5)\end{array}$ & $\begin{array}{c}101 \\
(10.1)\end{array}$ & & $\begin{array}{c}666 \\
(10.5)\end{array}$ & $\begin{array}{c}559 \\
(10.5)\end{array}$ & $\begin{array}{l}100 \\
(10.1)\end{array}$ & & $\begin{array}{c}659 \\
(10.4)\end{array}$ & $\begin{array}{c}567 \\
(10.5)\end{array}$ & $\begin{array}{c}101 \\
(10.1)\end{array}$ & & $\begin{array}{c}668 \\
(10.4)\end{array}$ & $\begin{array}{c}443 \\
(10.2)\end{array}$ & $\begin{array}{c}72 \\
(10.4)\end{array}$ & & $\begin{array}{c}515 \\
(10.2)\end{array}$ \\
\hline \multirow{7}{*}{ Education } & Primary & $\begin{array}{l}1024 \\
(19.1)\end{array}$ & $\begin{array}{c}165 \\
(16.5)\end{array}$ & \multirow{7}{*}{$<0.001$} & $\begin{array}{l}1189 \\
(18.7)\end{array}$ & $\begin{array}{l}1021 \\
(19.2)\end{array}$ & $\begin{array}{c}163 \\
(16.4)\end{array}$ & \multirow{7}{*}{$<0.001$} & $\begin{array}{l}1184 \\
(18.7)\end{array}$ & $\begin{array}{l}1026 \\
(19)\end{array}$ & $\begin{array}{c}165 \\
(16.5)\end{array}$ & \multirow{7}{*}{$<0.001$} & $\begin{array}{l}1191 \\
(18.6)\end{array}$ & $\begin{array}{c}871 \\
(20.1)\end{array}$ & $\begin{array}{c}140 \\
(20.3)\end{array}$ & \multirow{7}{*}{0.001} & $\begin{array}{l}1011 \\
(20.1)\end{array}$ \\
\hline & Vocational & 1291 & 309 & & 1600 & 1282 & 308 & & 1590 & 1296 & 309 & & 1605 & 1043 & 215 & & 1258 \\
\hline & vocdt & (24) & (30.8) & & (25.1) & (24.1) & (31) & & (25.2) & $(24)$ & $(30.9)$ & & $(25)$ & $(24)$ & (31.1) & & (25) \\
\hline & Hioh school & 1817 & 398 & & 2215 & 1795 & 394 & & 2189 & 1839 & 396 & & 2235 & 1448 & 252 & & 1700 \\
\hline & Milgn scriool & (33.8) & (39.7) & & (34.8) & (33.7) & (39.6) & & (34.7) & (34) & (39.6) & & (34.9) & (33.4) & (36.5) & & (33.8) \\
\hline & Tor & 1236 & 130 & & 1366 & 1225 & 129 & & 1354 & 1247 & 130 & & 1377 & 975 & 84 & & 1059 \\
\hline & lertiary & (23) & (13) & & (21.4) & (23) & (13) & & (21.4) & (23.1) & (13) & & (21.5) & (22.5) & (12.2) & & (21.1) \\
\hline \multirow{9}{*}{$\begin{array}{l}\text { Marital } \\
\text { status }\end{array}$} & Maniod & 3110 & 535 & \multirow{9}{*}{0.077} & 3645 & 3084 & 534 & \multirow{9}{*}{$<0.001$} & 3618 & 3128 & 535 & \multirow{9}{*}{$<0.001$} & 3663 & 2545 & 366 & \multirow{9}{*}{$<0.001$} & 2911 \\
\hline & Married & (57.9) & (53.4) & & (57.2) & (57.9) & (53.7) & & (57.3) & (57.8) & (53.5) & & (57.2) & (58.7) & (53) & & (57.9) \\
\hline & Single & 1026 & 187 & & 1213 & 1016 & 183 & & 1199 & 1040 & 186 & & 1226 & 709 & 100 & & 809 \\
\hline & & (19.1) & (18.7) & & (19) & (19.1) & (18.4) & & (19) & (19.2) & (18.6) & & (19.1) & (16.3) & (14.5) & & (16.1) \\
\hline & Divorced & 425 & 149 & & 574 & 423 & 147 & & 570 & 428 & 148 & & 576 & 354 & 104 & & 458 \\
\hline & & (7.9) & (14.9) & & (9) & $(7.9)$ & (14.8) & & (9) & (7.9) & (14.8) & & (9) & $(8.2)$ & (15.1) & & $(9.1)$ \\
\hline & Widowed & 684 & 126 & & 810 & 683 & 125 & & 808 & 685 & 126 & & 811 & 632 & 119 & & 751 \\
\hline & vviaumea & (12.7) & (12.6) & & (12.7) & (12.8) & (12.6) & & (12.8) & (12.7) & (12.6) & & (12.7) & (14.6) & (17.2) & & (14.9) \\
\hline & Missing & 123 & $5(0.5)$ & & 128 & 117 & $5(0.5)$ & & 122 & 127 & $5(0.5)$ & & 132 & 97 & $2(0.3)$ & & $99(2)$ \\
\hline
\end{tabular}




\begin{tabular}{|c|c|c|c|c|c|c|c|c|c|c|c|c|c|c|c|c|c|}
\hline & & $(2.3)$ & & & (2) & $(2.2)$ & & & (1.9) & $(2.3)$ & & & $(2.1)$ & $(2.2)$ & & & \\
\hline \multirow{3}{*}{ Ethnicity } & non-Roma & $\begin{array}{c}5251 \\
(97.8)\end{array}$ & $\begin{array}{c}937 \\
(93.5)\end{array}$ & \multirow{3}{*}{$<0.001$} & $\begin{array}{c}6188 \\
(97.1)\end{array}$ & $\begin{array}{c}5209 \\
(97.9)\end{array}$ & $\begin{array}{c}930 \\
(93.6)\end{array}$ & \multirow{3}{*}{$<0.001$} & $\begin{array}{c}6139 \\
(97.2)\end{array}$ & $\begin{array}{c}5291 \\
(97.8)\end{array}$ & $\begin{array}{c}935 \\
(93.5)\end{array}$ & \multirow{3}{*}{$<0.001$} & $\begin{array}{c}6226 \\
(97.2)\end{array}$ & $\begin{array}{c}4244 \\
(97.9)\end{array}$ & $\begin{array}{c}647 \\
(93.6)\end{array}$ & \multirow{3}{*}{$<0.001$} & $\begin{array}{c}4891 \\
(97.3)\end{array}$ \\
\hline & Roma & $\begin{array}{c}104 \\
(1.9)\end{array}$ & $\begin{array}{c}65 \\
(6.5)\end{array}$ & & $\begin{array}{l}169 \\
(2.7)\end{array}$ & $\begin{array}{c}102 \\
(1.9)\end{array}$ & $\begin{array}{c}64 \\
(6.4)\end{array}$ & & $\begin{array}{l}166 \\
(2.6)\end{array}$ & $\begin{array}{c}104 \\
(1.9)\end{array}$ & $\begin{array}{c}65 \\
(6.5)\end{array}$ & & $\begin{array}{c}169 \\
(2.6)\end{array}$ & $87(2)$ & $\begin{array}{c}44 \\
(6.4)\end{array}$ & & $\begin{array}{c}131 \\
(2.6)\end{array}$ \\
\hline & Missing & $\begin{array}{c}13 \\
(0.2)\end{array}$ & (0) & & $\begin{array}{c}13 \\
(0.2)\end{array}$ & $\begin{array}{c}12 \\
(0.2)\end{array}$ & (0) & & $\begin{array}{c}12 \\
(0.2)\end{array}$ & $\begin{array}{c}13 \\
(0.2)\end{array}$ & (0) & & $\begin{array}{c}13 \\
(0.2)\end{array}$ & $6(0.1)$ & (0) & & $6(0.1)$ \\
\hline Total & & $\begin{array}{l}5368 \\
(100) \\
\end{array}$ & $\begin{array}{l}1002 \\
(100) \\
\end{array}$ & - & $\begin{array}{l}6370 \\
(100)\end{array}$ & $\begin{array}{l}5323 \\
(100)\end{array}$ & $\begin{array}{c}994 \\
(100) \\
\end{array}$ & - & $\begin{array}{l}6317 \\
(100)\end{array}$ & $\begin{array}{l}5408 \\
(100) \\
\end{array}$ & $\begin{array}{l}1000 \\
(100)\end{array}$ & _. & $\begin{array}{l}6408 \\
(100) \\
\end{array}$ & $\begin{array}{l}4337 \\
(100) \\
\end{array}$ & $\begin{array}{c}691 \\
(100) \\
\end{array}$ & & $\begin{array}{l}5028 \\
(100) \\
\end{array}$ \\
\hline
\end{tabular}

"Chi-square test for pre-pandemic and pandemic periods between groups 


\subsection{Outcome measures in the prepandemic and the COVID-19 pandemic periods}

There were 4251 and 561 subjects who visited GPs within a year, corresponding to a prevalence of $79.2 \%$ (95\% CI 78.1-80.3) and $56 \%$ (95\% CI 52.9-59.1) in the prepandemic and pandemic periods, respectively. The number of participants who visited a specialist in a year was 3426 in the prepandemic period (prevalence of $64.4 \%, 95 \% \mathrm{CI} 63.1-65.7$ ) and 378 in the pandemic period (prevalence of $38.0 \%, 95 \%$ CI 35.0-41.0). A total of 728 and 68 subjects were admitted to the hospital a year before (prevalence of $13.5 \%$, 95\% CI 12.6-14.4) and during the pandemic (prevalence of 6.8\%, 95\% CI 5.2-8.4), respectively. These outcomes were reduced significantly during the pandemic period. Because CRPNR was faced by 245 and 36 participants, the prepandemic (5.6\%, 95\% CI 4.9-6.3) and pandemic (5.2\%, 95\% CI 3.5-6.9) prevalence did not differ.

\subsubsection{Factors associated with health care use and the role of the COVID-19 pandemic}

Older age, female sex, and the prevalence of a chronic disease correlated with more intensive use of GPs, specialists, and hospital care but seemed to be independent of the CRPNR. Patients with COPD, IHD or diabetes faced CRPNR more frequently. Regional inequalities were observed for each outcome.

A higher level of education was associated with more intensive use of GPs and specialist care, as well as with less frequent hospital admissions and CRPNR. Roma people use GPs and specialist care more frequently, and they face CRPNR more often than non-Roma people do. The occurrence of each outcome was more frequent among widowed patients and less frequent (apart from the CRPNR) among single patients. The probability of CRPNR was significantly elevated among divorced patients. (Table 2) Detailed descriptive measures for each outcome in both study periods by population strata are summarized in Appendix Tables S1-4.

Table 2. Change of the health service use in COVID-19 pandemic period compared to prepandemic period in different social strata.

\begin{tabular}{|c|c|c|c|c|c|}
\hline & Character & tics & $\begin{array}{l}\text { Prepandemic } \\
\text { prevalence* }\end{array}$ & Pandemic prevalence* & OR $(95 \% C I)^{* *}$ \\
\hline \multirow{12}{*}{ 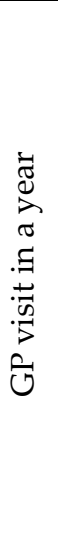 } & \multirow{4}{*}{$\begin{array}{l}\text { Educatio } \\
\text { n level }\end{array}$} & Primary & $827(80.8)$ & $121(73.3)$ & reference \\
\hline & & Vocational & $1026(79.5)$ & $176(57.0)$ & $0.768(0.641-0.920)$ \\
\hline & & High school & $1456(80.1)$ & $200(50.3)$ & $0.753(0.635-0.893)$ \\
\hline & & Tertiary & $942(76.2)$ & $64(49.2)$ & $0.710(0.590-0.855)$ \\
\hline & \multirow{5}{*}{$\begin{array}{l}\text { Marital } \\
\text { status }\end{array}$} & Married & $2472(79.5)$ & $295(55.1)$ & reference \\
\hline & & Single & $727(70.9)$ & $72(38.5)$ & $0.612(0.532-0.705)$ \\
\hline & & Divorced & $345(81.2)$ & $76(51.0)$ & $0.873(0.715-1.066)$ \\
\hline & & Widowed & $621(90.8)$ & $116(92.1)$ & $3.204(2.489-4.122)$ \\
\hline & & Missed & $86(69.9)$ & $2(40.0)$ & $\mathrm{NC}$ \\
\hline & \multirow{3}{*}{ Ethnicity } & non-Roma & $4167(79.4)$ & $529(56.5)$ & reference \\
\hline & & Roma & $76(73.1)$ & $32(49.2)$ & $0.563(0.409-0.774)$ \\
\hline & & Missed & $8(61.5)$ & $0(0.0)$ & $\mathrm{NC}$ \\
\hline \multirow{11}{*}{ 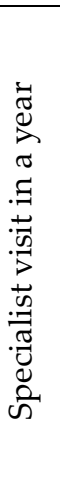 } & \multirow{4}{*}{$\begin{array}{l}\text { Educatio } \\
\text { n level }\end{array}$} & Primary & $614(60.1)$ & $84(51.5)$ & reference \\
\hline & & Vocational & $790(61.6)$ & $115(37.3)$ & $0.920(0.790-1.071)$ \\
\hline & & High school & 1177 (65.6) & $145(36.8)$ & $1.062(0.919-1.226)$ \\
\hline & & Tertiary & $845(69.0)$ & $34(26.4)$ & $1.288(1.097-1.513)$ \\
\hline & \multirow{5}{*}{$\begin{array}{l}\text { Marital } \\
\text { status }\end{array}$} & Married & $2054(66.6)$ & $203(38.0)$ & reference \\
\hline & & Single & $533(52.5)$ & $42(23.0)$ & $0.556(0.487-0.634)$ \\
\hline & & Divorced & $293(69.3)$ & 49 (33.3) & $0.905(0.755-1.084)$ \\
\hline & & Widowed & $475(69.5)$ & $82(65.6)$ & $1.338(1.136-1.576)$ \\
\hline & & Missed & $71(60.7)$ & $2(40.0)$ & $\mathrm{NC}$ \\
\hline & \multirow{2}{*}{ Ethnicity } & non-Roma & $3372(64.7)$ & $354(38.1)$ & reference \\
\hline & & Roma & $47(46.1)$ & $24(37.5)$ & $0.484(0.354-0.661)$ \\
\hline
\end{tabular}




\begin{tabular}{|c|c|c|c|c|c|}
\hline & & Missed & $7(58.3)$ & $0(0.0)$ & $\mathrm{NC}$ \\
\hline \multirow{12}{*}{ 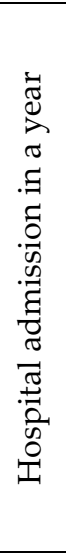 } & \multirow{4}{*}{$\begin{array}{l}\text { Educatio } \\
\text { n level }\end{array}$} & Primary & $199(19.4)$ & $22(13.3)$ & reference \\
\hline & & Vocational & $182(14)$ & $24(7.8)$ & $0.646(0.526-0.795)$ \\
\hline & & High school & $207(11.3)$ & $17(4.3)$ & $0.489(0.400-0.598)$ \\
\hline & & Tertiary & $140(11.2)$ & $5(3.8)$ & $0.517(0.412-0.647)$ \\
\hline & \multirow{5}{*}{$\begin{array}{l}\text { Marital } \\
\text { status }\end{array}$} & Married & $404(12.9)$ & $31(5.8)$ & reference \\
\hline & & Single & $87(8.4)$ & $9(4.8)$ & $0.630(0.500-0.794)$ \\
\hline & & Divorced & $71(16.6)$ & $9(6.1)$ & $1.197(0.926-1.547)$ \\
\hline & & Widowed & $150(21.9)$ & $19(15.1)$ & $1.953(1.604-2.378)$ \\
\hline & & Missed & $16(12.6)$ & $0(0.0)$ & $\mathrm{NC}$ \\
\hline & \multirow{3}{*}{ Ethnicity } & non-Roma & $705(13.3)$ & $62(6.6)$ & reference \\
\hline & & Roma & $20(19.2)$ & $6(9.2)$ & $1.294(0.846-1.979)$ \\
\hline & & Missed & $3(23.1)$ & $0(0.0)$ & $\mathrm{NC}$ \\
\hline \multirow{12}{*}{ 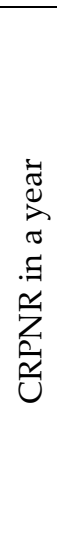 } & \multirow{4}{*}{$\begin{array}{l}\text { Educatio } \\
\text { n level }\end{array}$} & Primary & $83(9.5)$ & $23(16.4)$ & reference \\
\hline & & Vocational & $49(4.7)$ & $8(3.7)$ & $0.405(0.290-0.565)$ \\
\hline & & High school & $81(5.6)$ & $5(2.0)$ & $0.455(0.338-0.612)$ \\
\hline & & Tertiary & $32(3.3)$ & $(0.0)$ & $0.266(0.177-0.399)$ \\
\hline & \multirow{5}{*}{$\begin{array}{l}\text { Marital } \\
\text { status }\end{array}$} & Married & $126(5.0)$ & $10(2.7)$ & reference \\
\hline & & Single & $44(6.2)$ & $4(4.0)$ & $1.287(0.917-1.806)$ \\
\hline & & Divorced & $25(7.1)$ & $9(8.7)$ & $1.636(1.108-2.415)$ \\
\hline & & Widowed & $44(7.0)$ & $13(10.9)$ & $1.676(1.217-2.308)$ \\
\hline & & Missed & $6(6.2)$ & $0(0.0)$ & $\mathrm{NC}$ \\
\hline & \multirow{3}{*}{ Ethnicity } & non-Roma & $230(5.4)$ & $28(4.3)$ & reference \\
\hline & & Roma & $14(16.1)$ & $8(18.2)$ & $3.624(2.254-5.828)$ \\
\hline & & Missed & $1(16.7)$ & $0(0.0)$ & $\mathrm{NC}$ \\
\hline
\end{tabular}

* Number of cases (and proportion as \%) of positive outcomes

** odds ratios with $95 \%$ confidence intervals from logistic regression models

NC- not computable

\subsection{Determinants of health care use and the subgroup-specific effect of the pandemic}

The most intensive decline was observed in the probability of specialist visits and hospital admissions in the year during the pandemic. The decline in GP visit frequency was weaker and proved to be borderline significant in the multivariable model. The CRPNR showed no change during the pandemic period (Table 3).

In the multivariable logistic regression models, older age, female sex, and chronic disease proved to be factors associated with more use of health care services. There was significant geographical variability in health care use as well.

Health care use followed the general pattern among Roma: Roma ethnicity did not show a significant impact on GP visits, specialist visits, or hospital visits. However, CRPNR was more frequent $(\mathrm{aOR}=2.018$, 95\% CI: 1.061-3.838). Marital status was independent of the studied outcomes apart from the specialist visits in a year, which was less frequent among single (aOR=0.753, 95\% CI: 0.636-0.891) and widowed (aOR=0.740, 95\% CI: 0.597-0.918) patients. Although the role of higher-level education as a determinant of more frequent use of specialist care, less frequent hospital admissions and less frequent experience with CRPNR was confirmed by a complex model and the GP visits in a year proved to be more frequent among more educated participants after controlling for the sociodemographic and clinical status of the survey participants. (Table 3 ).

The uneven distribution of the pandemic effect by socioeconomic status was established by multivariable models. The pandemic decline in hospital admission was evenly distributed by education subgroups. The pandemic effect was stronger among more ed- 
ucated individuals with respect to GP (iORhigh school/primary $=0.434,95 \%$ CI: $0.243-0.776$ ) and specialist visit (iORhigh school/primary $=0.598,95 \%$ CI: $0.364-0.985$, iORtertiary/primary $=0.331,95 \%$ CI: $0.179-0.611)$ frequency. Additionally, the higher the education, the lower the CRPNR (iORhigh school/primary $=0.236,95 \%$ CI: $0.075-0.743$ ) as shown in the multivariable model. Considering marital status, among widowed women, general declines in GP visit (iOR=2.284, 95\% CI: 1.043-4.998) and specialist visit (iOR=1.915, 95\% CI: 1.157-3.168) frequencies were not manifested and were counterbalanced. Other significant interactions of marital status with the pandemic were not established by the applied models. Because a significant interaction between Roma ethnicity and time was not confirmed in the multivariable approach, the Roma and non-Roma were no different with respect to pandemic reactions in terms of health care use (Table 3).

Table 3. Determinants of health care use by multivariable logistic regression models (by odds ratios with 95\% confidence intervals) controlled for the interaction between pandemic and studied sociodemographic characteristics.

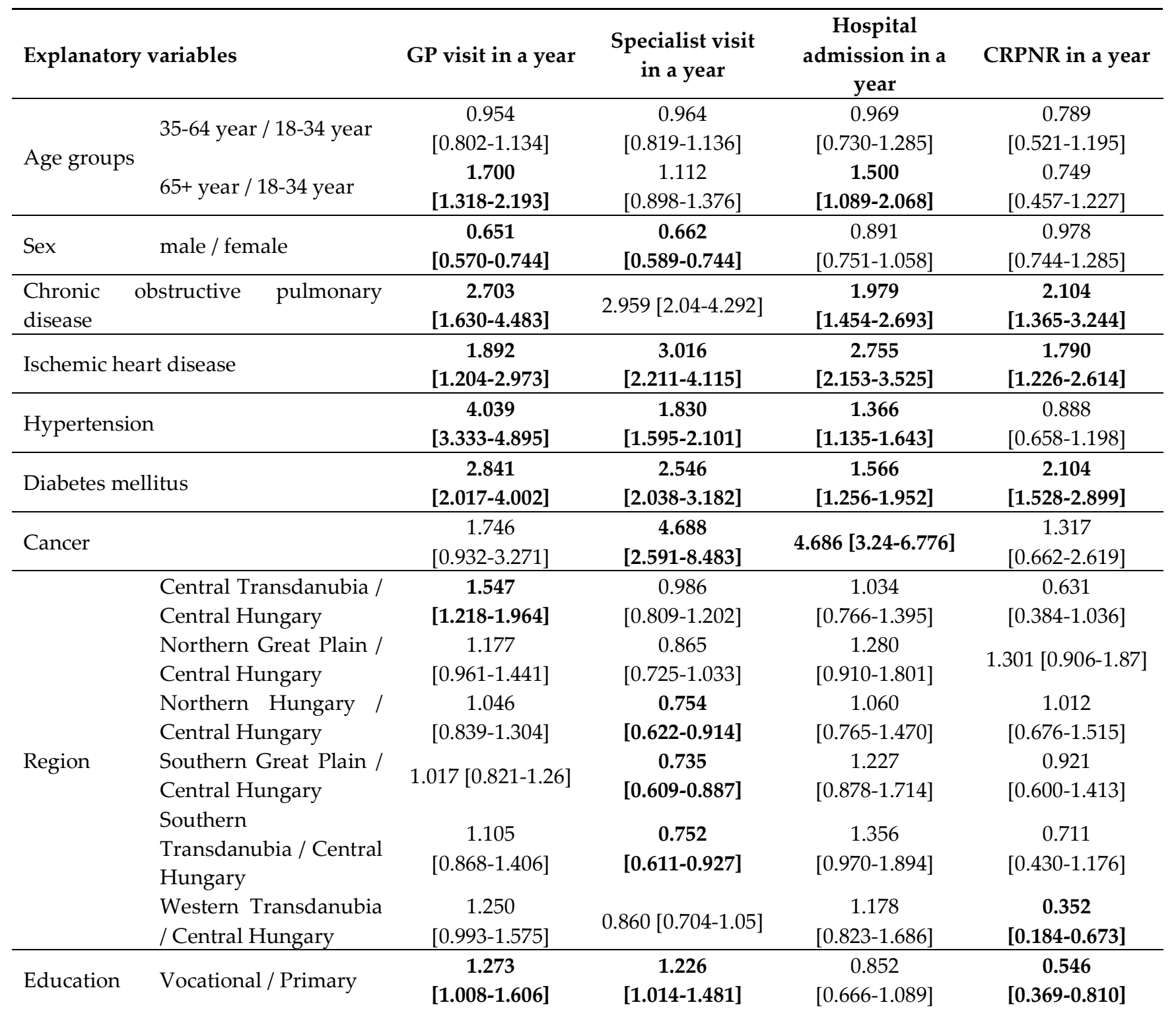




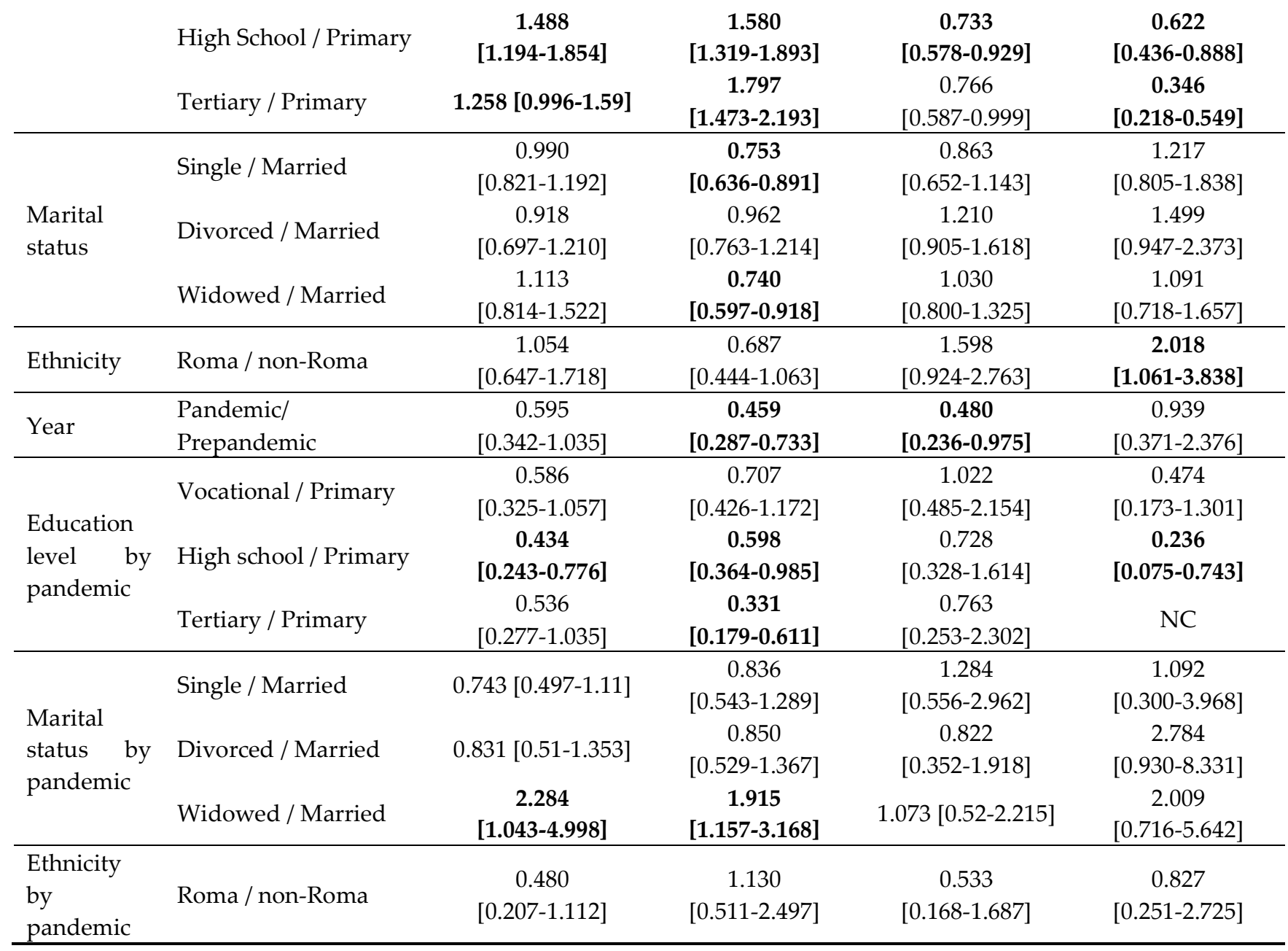




\section{Discussion}

\subsection{Main findings}

Through this population-based cross-sectional study, we investigated the effect of the COVID-19 pandemic on health care use in Hungary. Data analysis demonstrated a profound pandemic decrease in the GP visit frequency, specialist visit frequency, and hospital admission rate (by $23.2 \%, 26.4 \%$, and $6.7 \%$, respectively) but not in the occurrence of CRPNR when comparing the prepandemic and pandemic situations. According to publications about the use of specific health care facilities $[10,28,42-45]$ or on general health care access $[45,46]$, there was wide variability across European countries, and the Hungarian findings corresponded to the average of the European observations.

Hungary's prepandemic CRPNR was within the range of published references from developed countries [47]. The observed lack of change in the pandemic period in Hungary cannot be evaluated comparatively because there are no published pandemic CRPNR results from other countries.

Our results showed that older age, female sex, and chronic disease were associated with more use of health care services, and there were geographical inequalities in health care use. Considering social status, according to our multivariable models, the vulnerable groups are the primary educated, single, widowed, and Roma adults. These Hungarian observations are well supported in the international literature $[48,49]$. Altogether, the influence of sociodemographic and clinical status on health care use in Hungary has not deviated from the European mainstream.

\subsubsection{Pandemic impact by level of education}

The higher hospital admission rate of adults with primary-level education determined by our multivariable model, which was not changed during the pandemic, can be explained by their worse health status. Therefore, the observed higher frequency of GP and specialist visits among highly educated individuals cannot be explained by their poor health status and higher health care needs. Certainly, this inequality is a reflection of their differential ability and intention to use the existing services. This can be attributed to the higher proportion of nonurgent, elective actions in the medical intervention pattern of more educated individuals. This gap has been significantly narrowed during the pandemic period, suggesting that elective interventions were mainly postponed. These Hungarian observations are not in line with the independence between education and health care use during the pandemic, as described in the EU [29] and in the Netherlands [50], but these results were similar to the main findings of the SHARE Corona Survey on 27 European countries' 50+ year-old population [45]. Furthermore, our investigation demonstrated the inverse relationship of CRPNR with education, which was exaggerated in the pandemic period. Consequently, this gap has been widened.

\subsubsection{Pandemic impact among Roma}

The GP visit frequency was not associated with Roma ethnicity, and this relationship was not changed in the pandemic period. On the other hand, there were fewer outpatient specialist visits, and the hospital admission rate was higher among Roma patients, with a borderline significant difference. This shows that Roma individuals face serious limitations in secondary care access and that their health status is worse than that of non-Roma individuals. The CRPNR was significantly more frequent among Roma individu- 
als, reflecting that they are overrepresented among seriously deprived individuals in Hungary. Neither of these Roma-related inequalities changed significantly during the pandemic.

A limited access to outpatient specialists and a higher hospital admission rate of Roma has been demonstrated previously in Hungary [51], and this observation is similar to numerous reports from other countries [51-57]. Mainly based on these former experiences and on the demonstrated increased vulnerability of racial/ethnic minorities during the COVID-19 pandemic [58], adequate monitoring among the Roma to avoid unequal health service delivery is recommended [59-61]. However, our investigation could not demonstrate the pandemic impact on ethnic inequalities in Hungary.

\subsubsection{Pandemic impact by marital status}

There was no inequality by marital status with respect to hospital admission and CRPNR in Hungary, and it was not changed during the pandemic.

In the crude analyses, the GP and specialist visit frequencies were the highest among widowed individuals, which could be attributed to the lack of informal care provided by the partner, which could have prevented the use of health services [62-64].

In the multivariable analyses controlled for the presence of chronic diseases, the widowed showed restricted access to specialists' care, suggesting that their needs are not being met properly.

The general reduction in GP and specialist access during the pandemic was not manifested at all among the widowed. The interaction analysis confirmed that widows were protected against the GP and specialist access restriction; that is, the special needs of the widowed were met in the pandemic period, resulting in a narrowing of marital status inequality.

\subsection{Practical Implications}

Pandemic health care restructuring to ensure the capacities for COVID-19 patients was inevitably accompanied by serious limitations in general health care availability. According to our analysis, the impact of these restrictions on hospital use was evenly distributed across the social subgroups. However, restrictions on GPs' and outpatient specialists' availability did unevenly affect the social strata. Most of the observed interactions between the social status and the pandemic can be considered adequate adaptations from the viewpoint of social equity, since the prepandemic inequalities, which discriminated the disadvantageous groups, were reduced in the pandemic period. The GPs' and outpatient specialists' visit reductions among highly educated adults (probably due to postponement of elective medical interventions), and the nondecreased GP and hardly decreased specialist visit frequency among the widowed reduced their disadvantageous status observed in the prepandemic period. The only inequality-increasing pandemic effect was observed among less educated individuals who faced more CRPNR than before the pandemic period. Altogether, health care access restraints were implemented by proper control for social consequences. The only gap widening the pandemic effect was the CRPNR increase among less educated individuals, which should be mitigated in the next phase of the epidemic.

4.3 Strengths and limitations 
In organizing this investigation, relevant questions on the EHIS were added to the ISSP questionnaire. It ensured the same structure of the datasets and the comparability of the two surveys' data.

In the merged database, the prepandemic reference period was represented by a fairly large sample, which resulted in the proper statistical power for evaluating the observations from the pandemic year.

There were statistically significant but practically hardly important differences in the social and clinical characteristics of the samples between the two study periods. Apart from the overrepresentation of middle-aged individuals in terms of GP visits, specialist visits and hospital admission datasets, the absolute differences in subgroup sharing were less than $10 \%$. Although our regression models were completed with sociodemographic and clinical variables, they could not compensate for the selection bias. Taking into consideration the small absolute differences in the representation and the strengths of the significant interactions in multivariable models, selection bias could not provide an alternative explanation for the main findings about the special sensitivity of certain social groups to the pandemic impact.

\section{Conclusions}

We investigated the social subgroup-specific effect of the COVID-19 pandemic on health care use in Hungary by a population-based cross-sectional study implemented before and during the pandemic. We demonstrated the manifestation of established sociodemographic inequalities in our sample by the level of education, marital status, and ethnicity. Our findings also show that (except for CRPNR) the COVID-19 pandemic drastically reduced the use of GP visits, outpatient specialist visits and the hospital admission rate in Hungary, consistent with the worldwide trend.

Roma proved to be not specifically affected by the pandemic in any studied respect. The pandemic restructuring of health care impacted the social subgroups evenly with respect to hospital care and unevenly with respect to GPs' and outpatient specialists' visits. Primary education and widowed did not follow the general trend, and their prepandemic limited health care use was not reduced further. This resulted in a pandemic-related inequality reduction. Supposing that postponing elective medical interventions - which dominated the advantageous and did not dominate the disadvantaged groups' medical intervention patterns-explains the gap reduction, this change corresponds to the intention of pandemic regulations.

The vulnerability of primary education to CRPNR was the only gap widened in the pandemic period. This shows that although the management of pandemic health care use restrictions was implemented to avoid social inequity in Hungary, the prevention of inequity in drug availability for primary educated individuals could require more support.

Supplementary Materials: Table S1: Stratum specific GP visit frequency in a year observed in prepandemic and pandemic periods; Table S2: Stratum specific specialist visit frequency in a year observed in prepandemic and pandemic periods; Table S3: Stratum specific hospital admission frequency in a year observed in prepandemic and pandemic periods; Table S4: Stratum specific CRPNR frequency in a year observed in prepandemic and pandemic periods. 
Author Contributions: Conceiving the design, data extraction, statistical analysis, interpretation, editing, B.B.B. and S.J.; reviewing, supervision, resources, project administration, S.J.; writing-original manuscript drafting, B.B.B.; B.A.A. and S.J; interpretation, reviewing the manuscript and final approval, B.B.B.; B.A.A.; R.N.W. and S.J.

Funding: The reported study was carried out in the framework of the " Routine monitoring for the health status and health care use in the Hungarian segregated colonies" program (BM/6327-3/2021, FEIF/951/2021-ITM), supported by the Deputy State Secretary for Social Inclusion of the Hungarian Government. In addition, the Stipendium Hungaricum Scholarship Program (grant SHE-15324-002/2018 to BBB and SHE-10324-004/2019 to RNW). Funders have no role in the design, collection, analysis, interpretations, and publication processes.

Institutional Review Board Statement: The EHIS wave 3 was endorsed by the European Statistical System Committee per Commission Implementing Regulation (EU) No. 255/2018. The ISSP data were used as per the Ethical statement of ISSP http://issp.org/fileadmin/user_upload/Rules_and_regulations/ethical_statement_issp.pdf the ethical requirements were approved according to the legal requirement of Hungary.

Informed Consent Statement: Not applicable.

Data Availability Statement: All information regarding the current study is available on the ISSP website unless the embargo period limits. While EHIS data is available from the Hungarian Central Statistical Office based on reasonable request.

Acknowledgments: We would like to thank all the study participants for providing their valuable time while collecting the data. We are also very grateful to Hungarian Central Statistical Office for supplying us with data. Stipendium Hungaricum Scholarship scheme is highly appreciated for granting an opportunity to achieve PhD studies for B.B.B. and R.N.W.

Conflicts of Interest: Authors declare that there is no competing interest.

Abbreviations: CI- confidence interval, COPD-Chronic obstructive pulmonary disease. COVID-19- coronavirus disease 19, CRPNR- cost-related prescription nonredemption, EHIS- European Health Interview Survey, EU- European Union, GP- General practitioner, IHD-Ischemic Heart Diseases, ISSP- International Social Survey Programme, OECD - Organization for Economic Cooperation and Development, OR- Odds ratio, PHC-Primary Health Care.

\section{References}

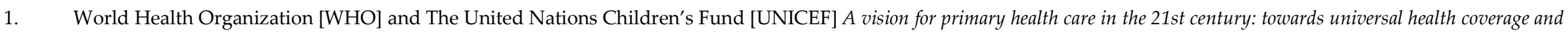
the Sustainable Development Goals.; Geneva, Switzerland, 2018; ISBN WHO/HIS/SDS/2018.X.

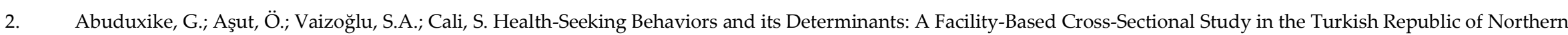
Cyprus. Int. J. Heal. Policy Manag. 2020, 9, 240-249, doi:10.15171/ijhpm.2019.106.

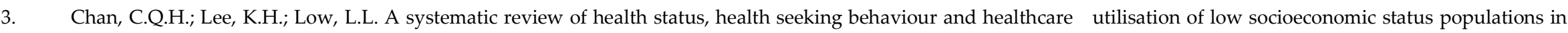
urban Singapore. Int. J. Equity Health 2018, 17, 39, doi:10.1186/s12939-018-0751-y.

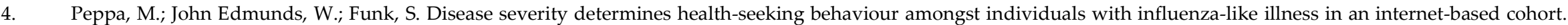


BMC Infect. Dis. 2017, 17, 238, doi:10.1186/s12879-017-2337-5.

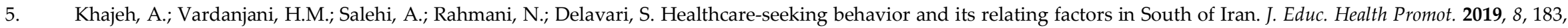
doi:10.4103/jehp.jehp_93_19.

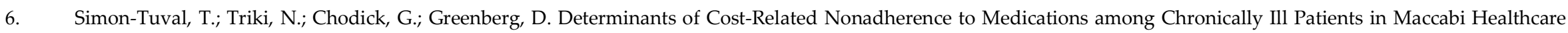
Services, Israel. Value Heal. Reg. Issues 2014, 4, 41-46, doi:https://doi.org/10.1016/j.vhri.2014.06.010.

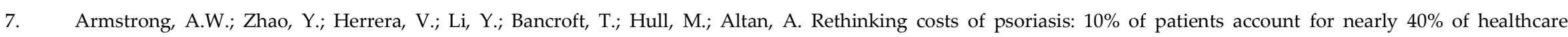
expenditures among enrollees with psoriasis in a U.S. health plan. J. Dermatolog. Treat. 2017, 28, 613-622, doi:10.1080/09546634.2017.1303566.

8. Golay, A. Pharmacoeconomic aspects of poor adherence: Can better adherence reduce healthcare costs? J. Med. Econ. 2011, 14, 594-608, doi:10.3111/13696998.2011.597808.

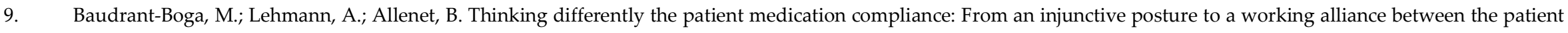
and the healthcare provider - Concepts and determinants [Penser autrement l'observance médicamenteuse: D'une posture injonctive à . Ann. Pharm. Fr. 2012, 70, 15-25, doi:10.1016/j.pharma.2011.10.003.

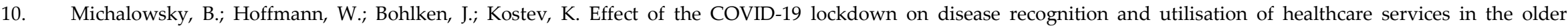
population in Germany: a cross-sectional study. Age Ageing 2020, 50, 317-325, doi:10.1093/ageing/afaa260.

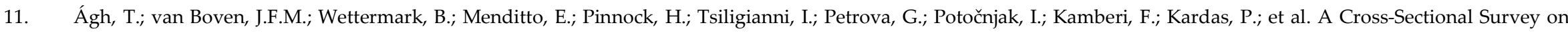
Medication Management Practices for Noncommunicable Diseases in Europe During the Second Wave of the COVID-19 Pandemic. Front. Pharmacol. 2021, 12, 1433, doi:10.3389/fphar.2021.685696.

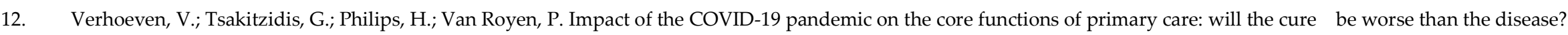
A qualitative interview study in Flemish GPs. BMJ Open 2020, 10, e039674, doi:10.1136/bmjopen-2020-039674.

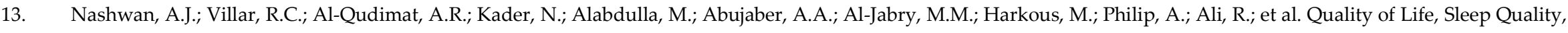
Depression, Anxiety, Stress, Eating Habits, and Social Bounds in Nurses during the Coronavirus Disease 2019 Pandemic in Qatar (The PROTECTOR Study): A Cross-Sectional, Comparative Study. J. Pers. Med. 2021, 11, doi:10.3390/jpm11090918.

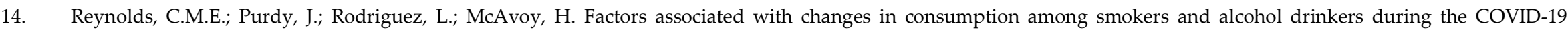
'lockdown' period. Eur. J. Public Health 2021, 31, 1084-1089, doi:10.1093/eurpub/ckab050.

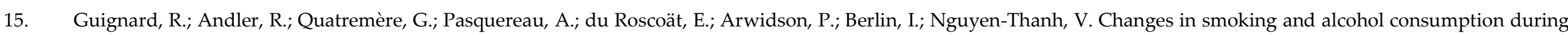
COVID-19-related lockdown: a cross-sectional study in France. Eur. J. Public Health 2021, 31, 1076-1083, doi:10.1093/eurpub/ckab054.

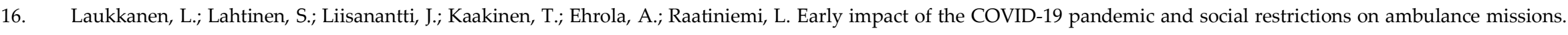
Eur. J. Public Health 2021, 31, 1090-1095, doi:10.1093/eurpub/ckab065. 


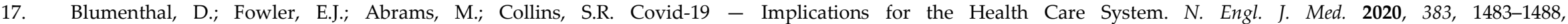
doi:10.1056/NEJMsb2021088.

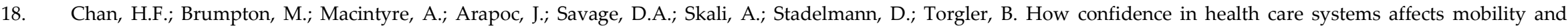
compliance during the COVID-19 pandemic. PLoS One 2020, 15, e0240644, doi:10.1371/journal.pone.0240644.

19. Mercier, G.; Arquizan, C.; Roubille, F. Understanding the effects of COVID-19 on health care and systems. Lancet Public Heal. 2020, 5, e524, doi:10.1016/S2468-2667(20)30213-9.

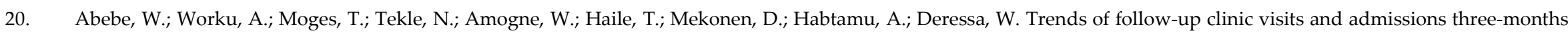
before and during COVID-19 pandemic at Tikur Anbessa specialized hospital, Addis Ababa, Ethiopia: an interrupted time series analysis. BMC Health Serv. Res. 2021, 21, 731, doi:10.1186/s12913-021-06730-8.

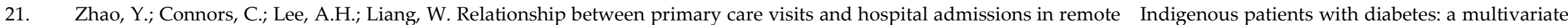
spline regression model. Diabetes Res. Clin. Pract. 2015, 108, 106-112, doi:10.1016/j.diabres.2015.01.013.

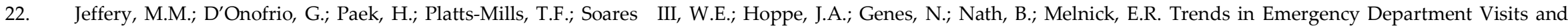
Hospital Admissions in Health Care Systems in 5 States in the First Months of the COVID-19 Pandemic in the US. JAMA Intern. Med. 2020, 180, 1328-1333, doi:10.1001/jamainternmed.2020.3288.

23. Hasen, G.; Edris, R.; Chala, G.; Tefera, Y.; Hussen, H.; Tekassa, T.; Suleman, S. Medicines dispensing practice during the era of COVID-19 pandemic: a commentary. J. Pharm. Policy Pract. 2021, 14, 1, doi:10.1186/s40545-020-00285-5.

24. Xiao, H.; Dai, X.; Wagenaar, B.H.; Liu, F.; Augusto, O.; Guo, Y.; Unger, J.M. The impact of the COVID-19 pandemic on health services utilization in China: Time-series analyses for 2016\&\#x2013;2020. Lancet Reg. Heal. - West. Pacific 2021, 9, doi:10.1016/j.lanwpc.2021.100122.

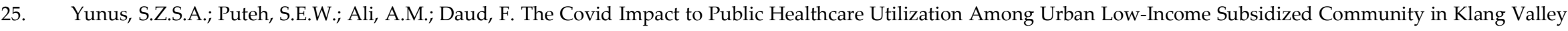
Malaysia. Heal. Serv. Res. Manag. Epidemiol. 2021, 8, 23333928211002410, doi:10.1177/23333928211002407.

26. SangNam Ahn, Kanghyock Koh, S.K. Changes in Healthcare Utilization, Spending, and Perceived Health during COVID - 19: A Longitudinal Study from Singapore Changes in Healthcare Utilization, Spending, and Perceived Health during COVID - 19: A Longitudinal Study from Singapore; 2020;

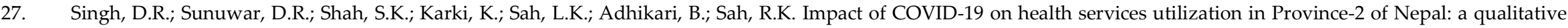
study among community members and stakeholders. BMC Health Serv. Res. 2021, 21, 174, doi:10.1186/s12913-021-06176-y.

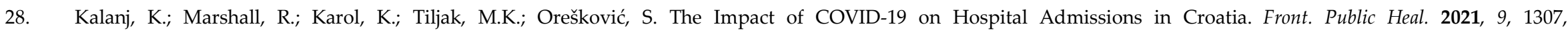
doi:10.3389/fpubh.2021.720948.

29. Ksinan Jiskrova, G.; Bobák, M.; Pikhart, H.; Ksinan, A.J. Job loss and lower healthcare utilisation due to COVID-19 among older adults across 27 European countries. J. 
Epidemiol. Community Health 2021, 75, 1078-1083, doi:10.1136/jech-2021-216715.

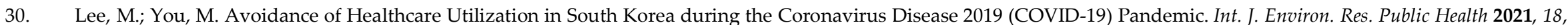
doi:10.3390/ijerph18084363.

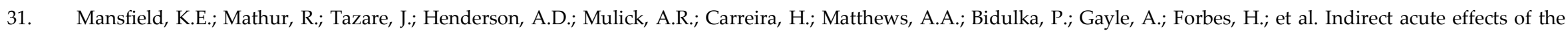
COVID-19 pandemic on physical and mental health in the UK: a population-based study. Lancet Digit. Heal. 2021, 3, e217-e230, doi:https://doi.org/10.1016/S2589-7500(21)00017-0.

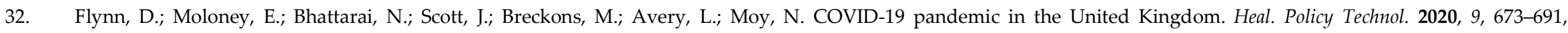
doi:10.1016/j.hlpt.2020.08.003.

33. European Union Agency for Fundamental Rights Coronavirus Pandemic in the EU - Impact on Roma and Travellers 2020.

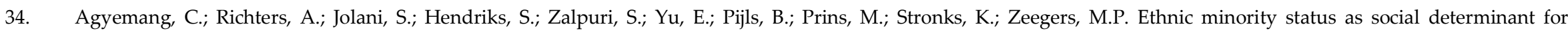
COVID-19 infection, hospitalisation, severity, ICU admission and deaths in the early phase of the pandemic: a meta-analysis. BMJ Glob. Heal. 2021, 6, doi:10.1136/bmjgh-2021-007433.

35. A3M Event page COVID-19 pandemic - Hungary Available online: https://www.global-monitoring.com/en/.

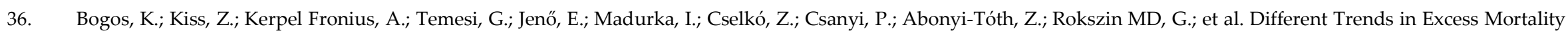
in a Central European Country Compared to Main European Regions in the Year of the COVID-19 Pandemic (2020): a Hungarian Analysis. Pathol. Oncol. Res. 2021, 27, 84, doi:10.3389/pore.2021.1609774.

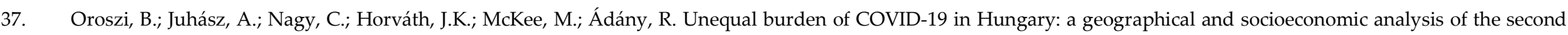
wave of the pandemic. BMJ Glob. Heal. 2021, 6, doi:10.1136/bmjgh-2021-006427.

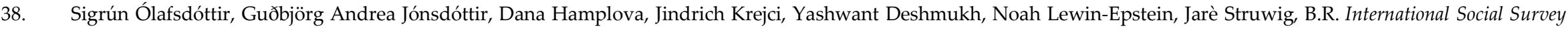
Programme 2021 Health and Health Care II;

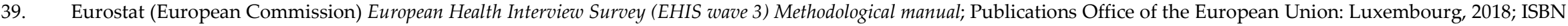
978-92-79-80333-8.

40. Lauer, S.R.; Yodanis, C.L. The International Social Survey Programme (ISSP): A Tool for Teaching with an International Perspective. Teach. Sociol. 2004, 32, 304-313.

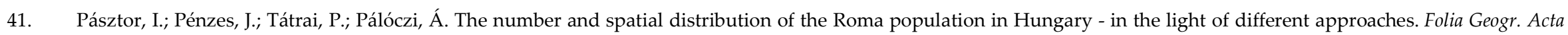
Fac. Stud. Humanit. naturae Univ. Prešoviensis, Prírodné vedy 2016, 58, 5-21.

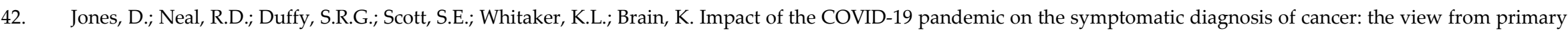
care. Lancet Oncol. 2020, 21, 748-750, doi:10.1016/S1470-2045(20)30242-4. 


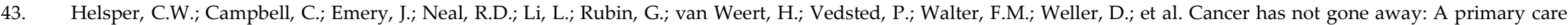
perspective to support a balanced approach for timely cancer diagnosis during COVID-19. Eur. J. Cancer Care (Engl). 2020, 29, e13290, doi:https://doi.org/10.1111/ecc.13290.

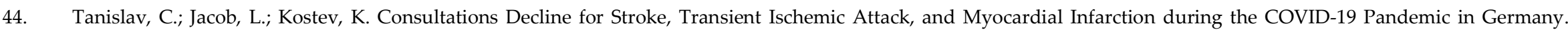
Neuroepidemiology 2021, 55, 70-78, doi:10.1159/000513812.

45. Smolić, Š.; Čipin, I.; Međimurec, P. Access to healthcare for people aged 50+ in Europe during the COVID-19 outbreak. Eur. J. Ageing 2021, doi:10.1007/s10433-021-00631-9.

46. World Health Organization Pulse survey on continuity of essential health services during the COVID-19 pandemic, Interim report 27 August 2020; Geneva, 2020;

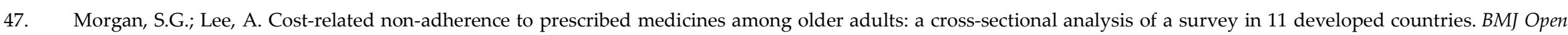
2017, 7, e014287, doi:10.1136/bmjopen-2016-014287.

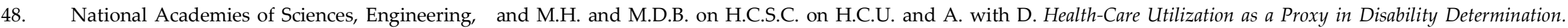
Washington (DC): National Academies Press (US); 2018 Mar 1; Washington (DC), 2018; ISBN 978-0-309-46918-0.

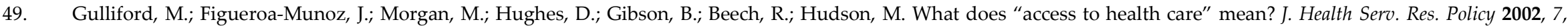
186-188, doi:10.1258/135581902760082517.

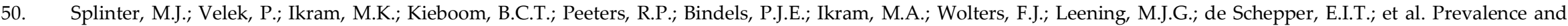
determinants of healthcare avoidance during the COVID-19 pandemic: A population-based cross-sectional study. PLOS Med. 2021, 18, 1-15, doi:10.1371/journal.pmed.1003854.

51. Pál, L.; Pálinkás, A.; Papp, M.; Sándor, J.; Falusi, Z.; Kovács, N.; Sipos, V.; Ádány, R.; Kőrösi, L.; Vincze, F.; et al. Healthcare Utilization and All-Cause Premature Mortality in Hungarian Segregated Roma Settlements: Evaluation of Specific Indicators in a Cross-Sectional Study. Int. J. Environ. Res. Public Health 2018, 15, 1835, doi:10.3390/ijerph15091835.

52. Jackson, C.; Bedford, H.; Cheater, F.M.; Condon, L.; Emslie, C.; Ireland, L.; Kemsley, P.; Kerr, S.; Lewis, H.J.; Mytton, J.; et al. Needles, Jabs and Jags: a qualitative exploration of barriers and facilitators to child and adult immunisation uptake among Gypsies, Travellers and Roma. BMC Public Health 2017, 17, 254, doi:10.1186/s12889-017-4178-y.

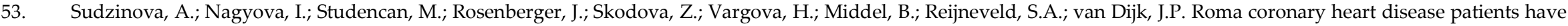
more medical risk factors and greater severity of coronary heart disease than non-Roma. Int. J. Public Health 2013, 58, 409-415, doi:10.1007/s00038-013-0462-5.

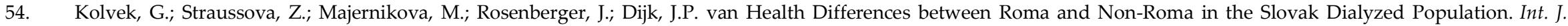
Environ. Res. Public Health 2018, 15, doi:10.3390/ijerph15020360.

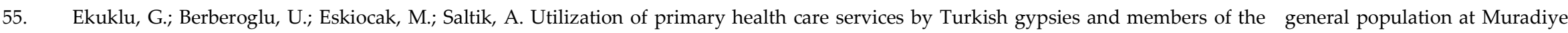
Health Unit District in Edirne, Turkey. Yonsei Med. J. 2003, 44, 414-423, doi:10.3349/ymj.2003.44.3.414.

56. Colombini, M.; Rechel, B.; Mayhew, S.H. Access of Roma to sexual and reproductive health services: Qualitative findings from Albania, Bulgaria and Macedonia. Glob. 
Public Health 2012, 7, 522-534, doi:10.1080/17441692.2011.641990.

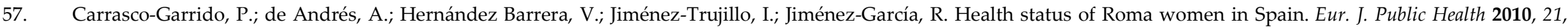
793-798, doi:10.1093/eurpub/ckq153.

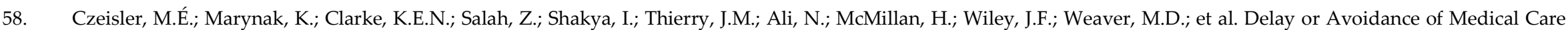
Because of COVID-19-Related Concerns - United States, June 2020. MMWR. Morb. Mortal. Wkly. Rep. 2020, 69, 1250-1257, doi:10.15585/mmwr.mm6936a4.

59. Korunovska, N.; Jovanovic, Z. ROMA IN THE COVID-19 CRISIS. An Early Warning from Six EU Member States; 2020;

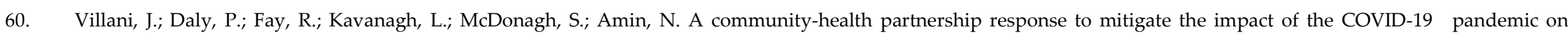
Travellers and Roma in Ireland. Glob. Health Promot. 2021, 28, 46-55, doi:10.1177/1757975921994075.

61. Kyriakides, S.; Dalli, H.; Schmit, N. EU Commissioners Outline COVID-19 Issues and Measures in a Letter to National Ministers. 2020.

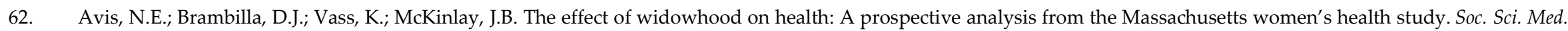
1991, 33, 1063-1070, doi:10.1016/0277-9536(91)90011-Z.

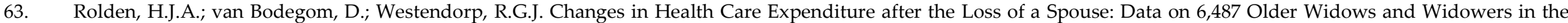
Netherlands. PLoS One 2014, 9, 1-17, doi:10.1371/journal.pone.0115478.

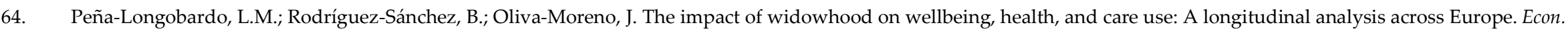
Hum. Biol. 2021, 43, 101049, doi:https://doi.org/10.1016/j.ehb.2021.101049. 